\title{
What do we need from catchment water quality models in Australia?
}

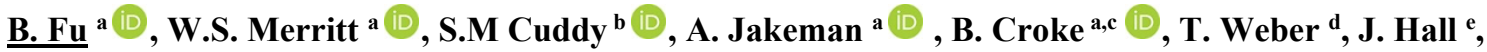 \\ D. Waters ${ }^{f}$ and D. Baker ${ }^{g}$
}

${ }^{a}$ Fenner School of Environment and Society, The Australian National University, ACT, Australia ${ }^{b}$ CSIRO Land \& Water, Canberra, ACT, Australia, ${ }^{c}$ Mathematical Sciences Institute, Australian National University, Australia. ${ }^{d}$ Alluvium, Australia. ${ }^{e}$ WA Department of Water and Environmental Regulation, Australia. ${ }^{f}$ Qld Department of Natural Resources, Mines and Energy, Australia. ${ }^{g}$ NSW Department of Primary Industries,

Australia.

Email: baihua.fu@anu.edu.au

\begin{abstract}
The published literature on the development and investigations into water quality modelling has primarily focused on making better predictions. Few studies have actively reported on model user needs and the implications of these needs on designing and selecting models that are fit-for-purpose. This paper describes the results of a Water Quality Model Needs survey that targeted persons developing or using models for catchment-scale Australian applications (Figure 1). Some 106 survey responses were received and analysed. Suspended sediment, total phosphorus and total nitrogen were highlighted by respondents as the most important constituents for catchment water quality models. The top five important drivers or management options were land use change, flow management, riparian management, climate change and point source control. Tool functionalities such as easy to include additional processes, output reporting, sensitivity analysis, and easy to access, use and learn were consistently identified as the most important features for catchment water quality models.
\end{abstract}

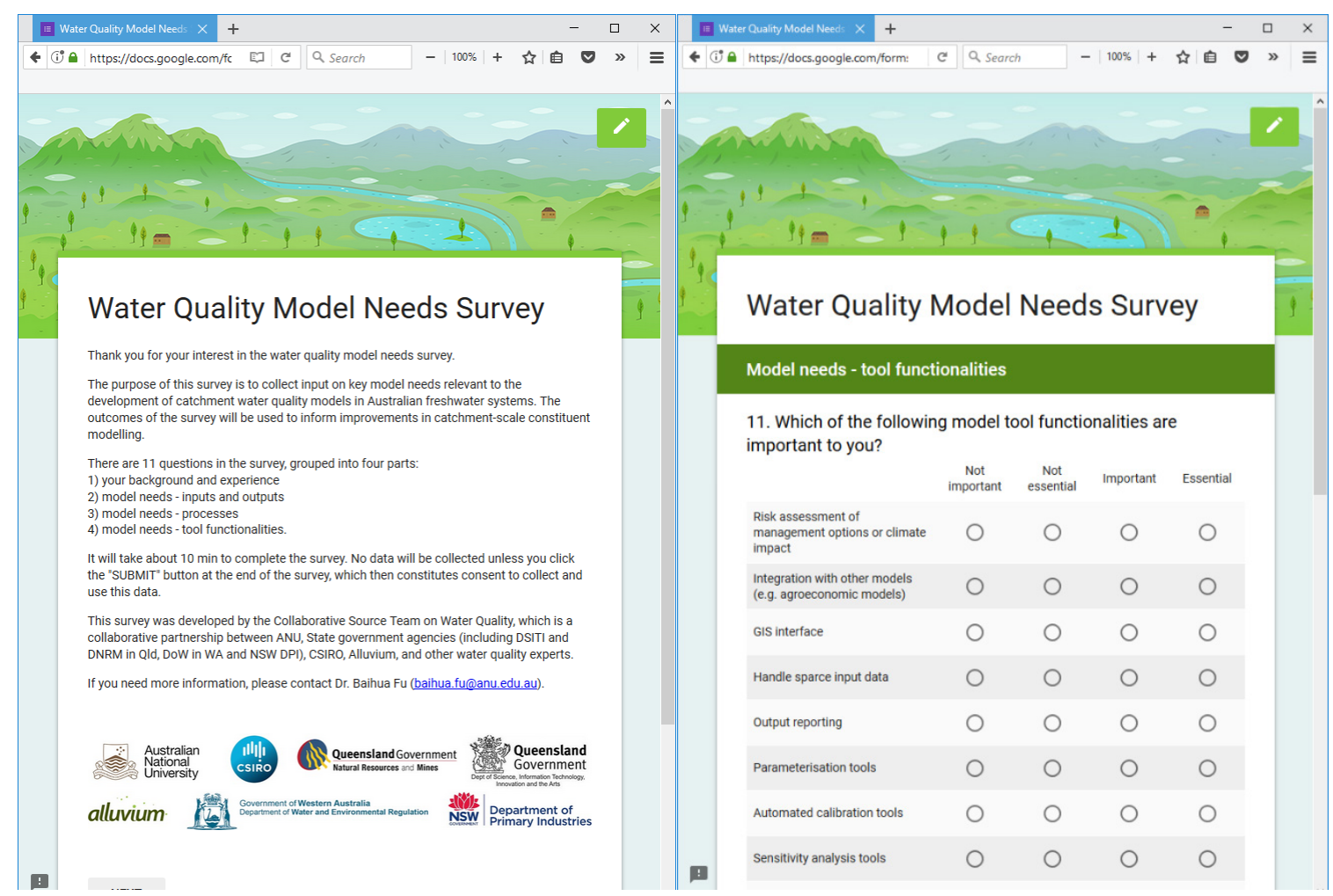

Figure 1. Screen shots of the online Google Forms survey

Keywords: Water quality modelling, catchment management, users, stakeholder needs 
Fu et al., What do we need from catchment water quality models in Australia?

\section{INTRODUCTION}

Many water quality models have been developed to determine the source, transformation and transport of constituents in catchments through a water body. In practice, they can be used to predict water quality in areas where monitoring is not feasible, or to predict water quality conditions resulting from different management strategies and under specified climate and/or land use change regimes. While water quality modelling studies have focused on making better predictions, few studies have reported on model user needs and how to design and select models that are fit-for-purpose. That is, a lot of focus has been on doing the sums right, but not a lot on doing the right sums.

Newham et al. (2004) conducted a survey of Australian water quality model user needs in late 2003. The survey was intended partly to inform the development of catchment water quality models in what is now the eWater Source software framework. In the last few years, Source has increasingly been adopted in Australia as a national level approach to catchment modelling, and large investments are being made by State and Federal government agencies to make the transition to the Source framework. However, it is our experience that the existing default water quality models in eWater Source do not have sufficient capacity to support investigation of the land use and land management changes happening, or proposed to happen, in Australian catchments. Considering it has been 15 years since the last model user needs survey (Newham et al. 2004), we re-visited the topic through a new survey in 2018 to ensure new model development aligns with current needs. The work was undertaken as part of the Improving Source Constituent Modelling Collaborative Partnership (thereafter the Partnership) that constitutes experts from ANU, State government agencies, CSIRO, and Alluvium. We report key findings of the survey which targeted persons developing or using water quality models for catchment-scale applications for Australian freshwater systems. The purpose of the survey was to elicit key model needs in inputs and outputs, process representations and tool functionalities. We also explored whether the questions that are now being asked by model developers or users are different to those asked in 2003 and whether or not there is a demand for water quality modelling with 'better' conceptualisation to meet the current needs.

\section{METHOD}

The 11 questions in the survey were grouped into four parts:

- Background and experience: to elicit each respondent's main activity, primary involvement with models, main interest in water quality models, modelling platform used, and general opinion on these models or platforms.

- Model needs in terms of inputs and outputs: to identify key constituents, drivers, types of temporal and spatial outputs, and output statistics to include in models.

- Model needs on processes: to identify important processes to represent in models.

- Model needs on tool functionalities: to rate the importance of specified model tool functionalities.

The survey design reflected the following considerations:

- Comprehensiveness: the survey covered a range of topics relating to model needs, including types of model inputs and outputs, processes involved and tool functionalities. Each question was designed to help us gain an improved understanding of model needs, and inform research directions in terms of prioritising actions to improve water quality models.

- Concise and unambiguous: the number of questions included in the survey was constrained such that the time required to complete the survey should be under 10 minutes. We took care to use unambiguous and intuitive questions that were relevant and that should be meaningful to respondents with different backgrounds or experience.

- Comparability to the earlier study: where feasible, we wanted to track if and how needs had changed over the years. Of the 14 questions in the Newham et al. (2004) study, eight relevant questions were included in this survey albeit with some modification. These covered the background and model inputs and outputs sections.

The survey was implemented and delivered using an online survey tool, Google Forms (Figure 1). Google Forms was selected because it is free and mobile-friendly. It offers the basic question types required for this survey, including text (e.g. for commenting), multiple choice, check boxes, grid (e.g. select important/not important for each option as demonstrated in the right-hand panel of Figure 1).

A draft survey was prepared and circulated among the Partnership. Once reviewed, eight members of the Partnership piloted the survey. The options for some of the questions were updated based on the answers and 
Fu et al., What do we need from catchment water quality models in Australia?

comments from this pilot survey, as well as learnings from additional review of literature undertaken by the authors of this paper (Fu et al. 2019).

Responses to the final survey were sought from practitioners, planners, researchers, policy makers and consultants in the fields of catchment management and water quality in Australia. The respondents include Australian members of the Modelling and Simulation Society of Australia and New Zealand Inc. (MSSANZ), science officers at the State government agencies that work on water quality modelling and/or management; and professional networks of the research team.

Some 106 survey responses were received during the survey period (December 2017 to January 2018) and used in the analysis. All responses to the survey were recorded using Google Forms. The data was exported to a csv spreadsheet. The R program (including base, reshape2, ggplot2, scales, likert, grid and plotly packages) was used to organise and analyse the survey data. Most of the analysis focused on counting the proportion of responses in each category.

\section{RESULTS}

\subsection{Background and experience}

Respondents were asked to identify their primary activity from a list of eight: academic research, science officer in government agencies, resource management, consulting, policy development, industry, community representation, or teaching. Only one option could be selected.

The largest group of respondents ( $>40 \%)$ was science officers in government agencies. The second largest respondent group was academic research, accounting for about $25 \%$ of the respondents. About $15 \%, 10 \%$ and $5 \%$ of respondents, respectively, identified their primary activity as being resource management, consulting, and policy development. Although the respondents in resource management and policy development were low in numbers, we expect that science officers in government agencies are well-connected with model users such as water or catchment managers and policy makers, and so their responses on the needs of water quality models for water management should align well with these two sub-groups.

Of the total respondents, $63 \%$ have been involved in the development of models and $57 \%$ use the results of modelling. About $20 \%$ were involved in the more technical aspect of modelling (e.g. calibration, evaluation and testing), the non-technical side (e.g. helping modellers in conceptualisation, provision of data support), or commission the development/use of the models. The smallest proportion (13\%) were involved in software or applications development.

The three most selected points of interest are water quality improvement, informing best management practices, and assessing the impact of land use changes. This highlights the need for modelling change due to management and/or land use, in addition to modelling the status of water quality. To model changes (e.g. using scenario analysis), it is important to capture the processes relating to the changes. This seems to be consistently felt by all groups of respondents, although those in academic research were strongly interested in climate change impacts and scientific enquiry in general. People in policy development reported strong interest in climate regional planning, river restoration and regulatory compliance. People in industries showed strong interest in monitoring and data collection, and urban development.

Reflecting its promotion as a national level approach to catchment modelling in Australian government agencies, eWater Source is the platform that the highest percentage of respondents $(25 \%)$ use on a routine basis; these respondents are typically science officers or consultants (Figure 2). The next most routinely used category was 'other' models $(22 \%)$, with similar numbers of science officers and academics selecting this option. This category includes a diverse range of models (e.g. SWMM, TUFLOW, RMA, LASCAM) and platforms (e.g. R, SQL, SAS, Matlab, Netica). About a third of the respondents sometimes use MUSIC, indicating their involvement in urban water quality modelling. Other models are not routinely used by the respondents, including SWAT which is extensively used in other countries.

Focusing on the model that a respondent mostly used, $39 \%$ of the respondents consider the model to meet their needs (Figure 3). This opinion is the most common view for users of the 'other' model category, reflecting these customised models are generally developed/selected in order to be fit-for-purpose. In contrast, those who mainly used Source most frequently agreed with the statement that 'the model needs to be improved by better capturing the management option at the right scale'. This statement also attracted $43 \%$ of all respondents. Some $26 \%$ of all respondents consider the models they mainly use to be too simple and missing key processes that they need to represent. And $21 \%$ of the respondents selected the statement on the lack of information on confidence levels in model outputs, a viewpoint that seems consistent across all surveyed models. Concerns 
Fu et al., What do we need from catchment water quality models in Australia?

relating to usability to non-technical people and run time were selected by $14 \%$ and $12 \%$ of the respondents, respectively. A small number of respondents (6.6\%) think the models they mainly use are too complex and that there is insufficient data to apply or calibrate the models. While we recognise that the answer to this question depends on the context of the applications users have been involved with, there does seem to be a stronger appetite among respondents for improving model representations and answering management questions than for improving model identifiability which aims to keep model complexity under control.

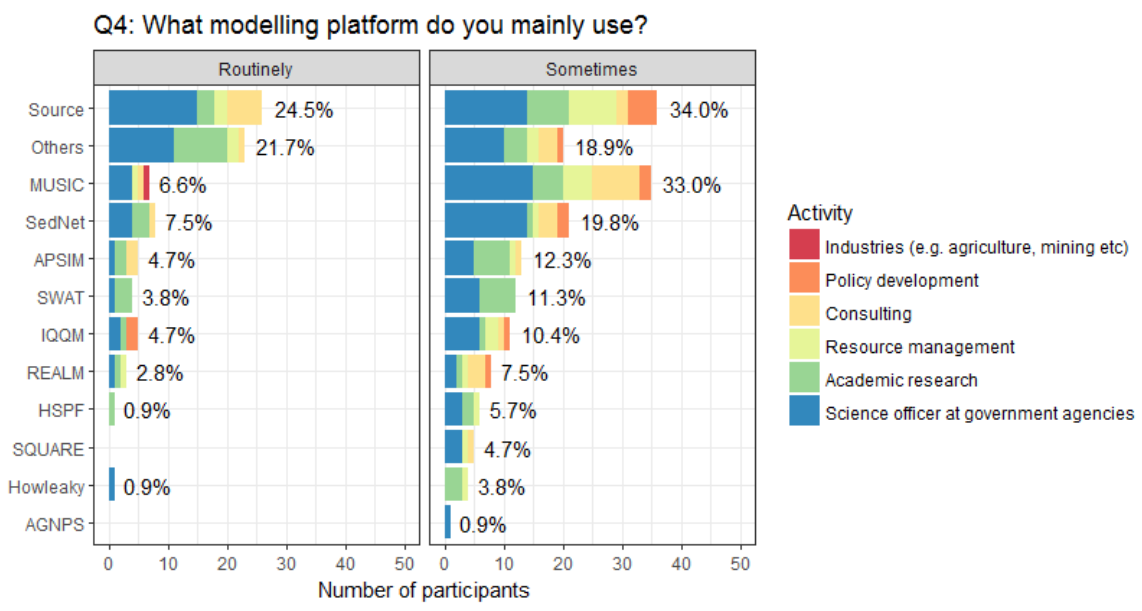

Figure 2. Main modelling platform used by respondents.

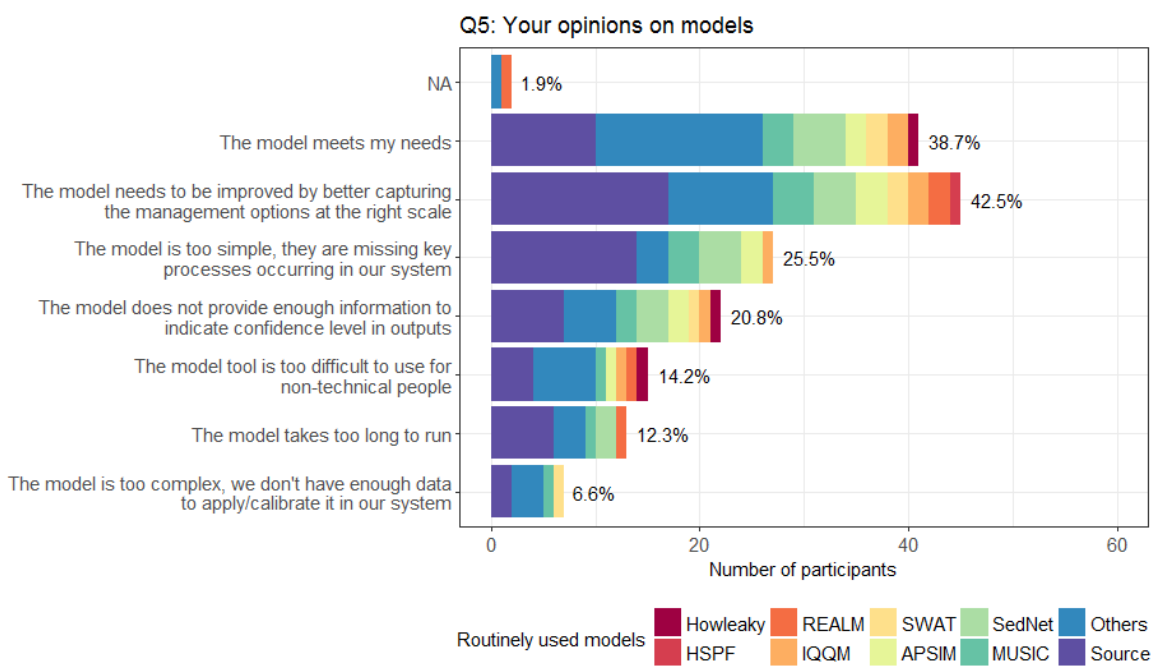

Figure 3. Respondent opinions on models they used most often, ordered by opinions.

\subsection{Model needs - inputs and outputs}

Respondents were asked to identify the constituents of most importance to them, either by selecting from the 14 listed constituents and/or identifying others. Over two thirds of respondents selected suspended sediment, total phosphorus and total nitrogen, and other species of nitrogen and phosphorus were selected by $38 \%$ to $46 \%$ of the respondents. Some $40 \%$ of the respondents considered salt as important, and $36 \%$ of the respondents considered pathogens as important.

With respect to including drivers or ameliorations/control options in a catchment water quality model, land use change was considered as very important by the greatest number of respondents, consistent across all groups of respondents (Figure 4). Other top drivers or management options include flow management, riparian management, climate change and point source control (Figure 4). Options such as tillage practices, groundwater pumping, planting and harvest operation were considered useful to include, but were ranked lower that other options. 
Fu et al., What do we need from catchment water quality models in Australia?

Q7: How useful is it to include the following drivers/options in a catchment water quality model?

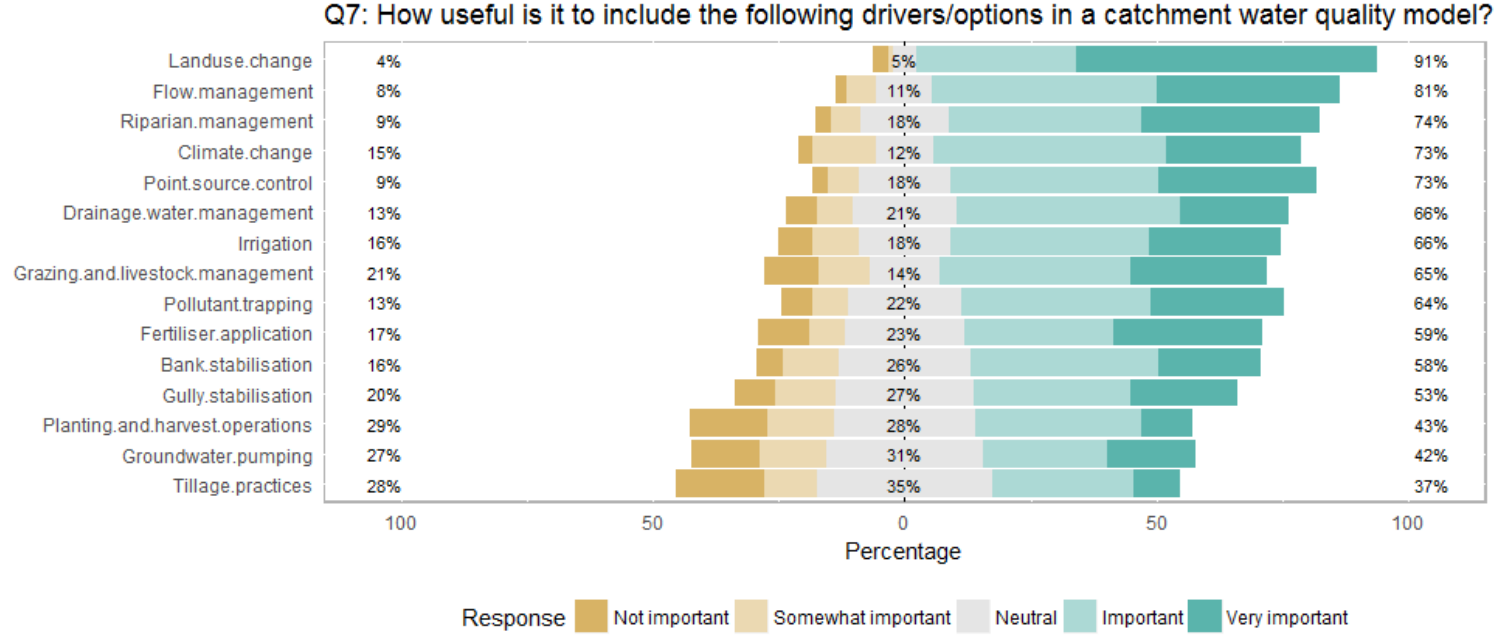

Figure 4. Likert chart on the importance of drivers or amelioration/control options in the catchment water quality models. The \% figures on the left are the percentages of respondents rated 'not important' or 'somewhat important', and the \% figures on the right are those rated 'important' or 'very important'.

By assigning numerical values for the options from 'not important' to 'very important' as 1 to 5, respectively, with an interval of 1 , we could calculate a mean and standard deviation importance rank for each of the options. Land use change scored the highest mean, 4.44, and lowest standard deviation, 0.87 (indicating high consistency in its ranking by all respondents). Flow management also scored a relatively high mean value (3.97) and low standard deviation (1.02). Options with more diverse answers were grazing and livestock management $(\mathrm{SD}=1.29)$, groundwater pumping $(\mathrm{SD}=1.26)$, and fertiliser application $(\mathrm{SD}=1.27)$.

Across the rating of importance in all drivers and management options, we found strong similarity in mean values between respondents whose main roles are in industries and in resource management (correlation $=$ 0.88 ), and between science officers and consulting (correlation $=0.84$ ), and resource management (correlation $=0.82$ ). Relatively low correlations were found between respondents from academic research and those in industries and policy development.

In terms of the temporal outputs, we found strong similarity between respondents in academic research, science officer and consulting, who identified daily concentrations and loads as the most useful temporal outputs. Annual and monthly loads were also considered useful. In comparison, daily and yearly temporal outputs were considered equally important for respondents in resource management. For those working in industries, annual outputs were more important than other temporal outputs. Mean or total loads or concentrations were considered by a majority of the respondents $(82 \%)$ as (one of) the most useful methods to aggregate time series data. This is consistent with the typical way in which aggregations are implemented in catchment water quality models.

The relative importance assigned to different spatial scales of model outputs was quite consistent amongst the respondent groups, bearing in mind the small size of some sub-groups. In terms of spatial scale of the model outputs, subcatchment aggregations were identified by $76 \%$ of the respondents, followed by catchment output $(67 \%)$ and stream reach (49\%). Some $33 \%$ of the respondents thought that spatially distributed (e.g. gridded) outputs were most useful.

\subsection{Model needs - processes}

The importance of process representation in a water quality model is strongly context dependant. That said, hydrology was identified by the greatest percentage of survey respondents (84\%) as most important in a water quality model; this was followed by catchment management (70\%), erosion and sediment generation (59\%), and nutrient cycling (52\%). Pesticide dynamics was the least nominated process $(12 \%)$, followed by plant growth (17\%), carbon dynamics (24\%), and ecological response dynamics (25\%).

There was general agreement in priority processes between the respondent groups, especially for the top five options. Some differences for options of lesser importance existed. For example, respondents in resource management were more likely to be interested in the cost-effectiveness of management options and trade-offs 
Fu et al., What do we need from catchment water quality models in Australia?

between water quality protection and other values, although this rated relatively low among academic researchers. In contrast to other groups, respondents in academic research showed relatively more concern around processes relating to lags in system response to management or pesticide dynamics, both of which are relatively poorly understood processes in the field of water quality.

\subsection{Model needs - tool functionalities}

Most of the model tool functionalities we included in the survey were considered either important or essential by the respondents (Figure 5). About $90 \%$ of the respondents considered the following functionalities to be either important or essential: easy to include additional processes, output reporting, sensitivity analysis tools and easy to access, use and learn. $77 \%$ to $82 \%$ of respondents thought it was important or essential to include uncertainty analysis tools, confidence or uncertainty reporting, risk assessment, parameterisation tools and handling sparse input data. Short run time was less frequently considered an important or essential functionality $(65 \%)$.

In general, respondents with different roles had similar opinions on the importance of tool functionalities, with some notable exceptions. Firstly, respondents in resource management were less likely to be concerned about operational features such as automated calibration, parameterisation, batch runs and run time in general, but were more likely to be concerned about output reporting, easy to use and learn, and handling sparse data. Respondents working in a policy development role exhibited some similarity to resource management, but showed stronger preferences for confidence or uncertainty reporting, GIS interface and risk assessment. Respondents in consulting were more likely to have stronger preference for batch runs, short run time, and integration with other models, but less concerned with confidence or uncertainty reporting or sensitivity and uncertainty analysis compared to other groups of respondents. Science officers and academic researchers showed similar interests in terms of tool functionalities, although academic researchers were more likely to be interested in easy to include additional processes, parameterisation tools, integration with other models, and being freely available.

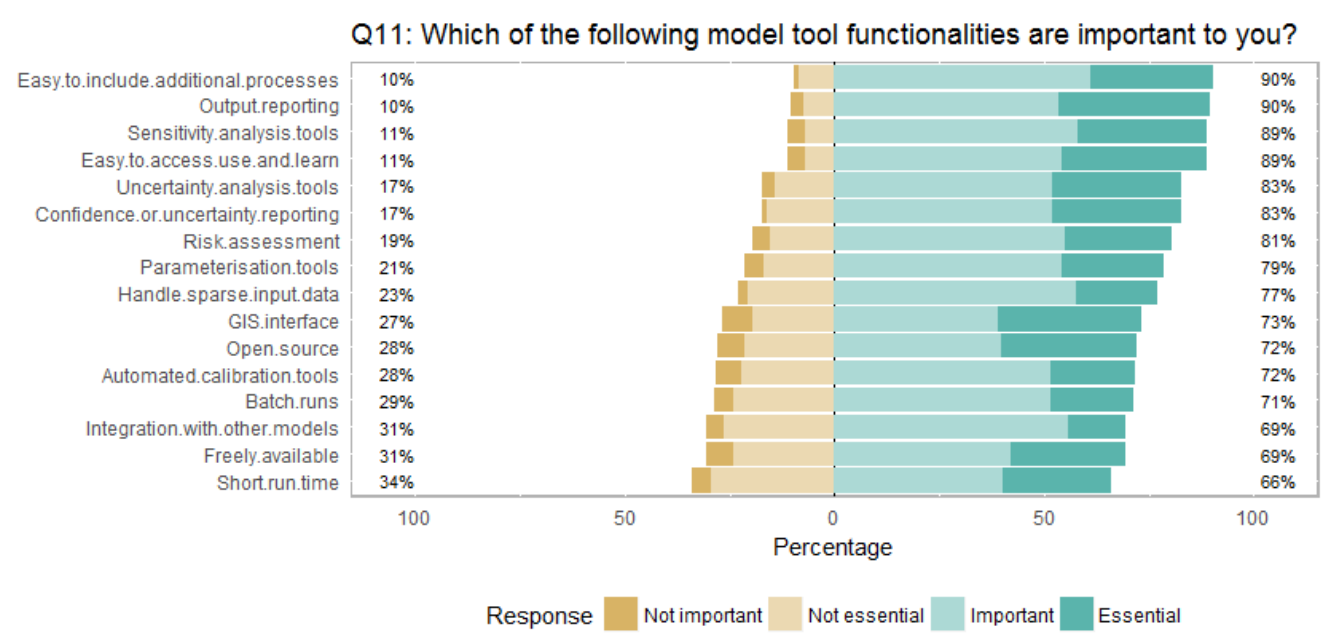

Figure 5. Likert chart indicating the importance of tool functionalities.

\section{SYNTHESIS}

Several key conclusions can be drawn from the survey:

1) Suspended sediment, total phosphorus and total nitrogen remain the most important constituents for catchment water quality models according to our surveyed group. The importance of salt and pesticides has dropped considerably compared to the 2003 survey (Newham et al., 2004), whilst the importance of speciation of nitrogen and phosphorus has increased slightly.

2) The top five important drivers or management options in the catchments models were land use change, flow management, riparian management, climate change and point source control. This is consistent with the findings from the 2003 survey. Other important management options included drainage management, irrigation, grazing and livestock management, pollutant trapping and fertiliser application. Groundwater pumping and tillage practices were ranked the lowest. 
3) A range of spatial and temporal scales were identified as important. The subcatchment scale remains the most useful spatial scale, but catchment outlet and reach scales were also useful. Daily scale remains the dominant temporal scale, but monthly and annual scales can also be useful, especially for those in resource management and industries.

4) Apart from hydrology, catchment management processes were considered the next most important to be included in catchment water quality models. Lack of data was considered as one of the most significant barriers to better incorporate management options in catchment water quality models.

5) The top four tool functionalities were: easy to include additional processes, output reporting, sensitivity analysis and easy to access, use and learn. This is consistent between participant groups.

As might be expected, eWater Source was reported as widely used within the Australian catchment water quality modelling community. There was a strong and consistent demand to improve the capability of water quality modelling in the eWater Source framework, particularly by incorporating better process representations and management options. Similar demands were expressed across the water quality modelling and management communities and for models other than eWater Source.

The 106 responses received for this survey is less than that obtained for the study by Newham et al. (2004), which attracted 176 completed responses. It is quite small considering the diversity of Australian environments from which we did not elicit information from respondents. Our respondents could be working in environments ranging from tropical or subtropical regions of Queensland to the inland arid lands of New South Wales to sandy ridges and plains in Western Australia, and many more. The results of this survey can only be seen as indicative although consistency shown between this survey and the 2003 survey provides some confidence that our results are likely to represent the opinions overall of the broader water quality modelling community.

Another cautionary note is that most respondents self-identified as either science officers (46) or academic research (26). Therefore, the results of the survey are biased towards the opinions expressed by these two groups and this is why our analysis focused mostly on comparing these two groups.

The actual needs in terms of model inputs, outputs, process representations and tool functionalities of a case study will depend on factors such as model purposes, characteristics of the study areas and data availability, and we acknowledge that the answers to some questions may be different depending on local context. However, the purpose of this survey was to identify general needs in water quality modelling for Australian freshwater systems, and we relied on the respondents to provide answers based on their most frequent and/or recent needs. Some respondents (e.g. those in consulting) may have broad needs due to their great range of activities, in which case many options could have been selected for a question where we restricted their options.

There has been increasing investments and activities in water quality models development in Australia in the last few years. Thus, it is suggested the survey may be re-visited in about 5-10 years. Future work may consider collecting and categorising interests by type of catchment. In addition, one-on-one interviews with selected respondents could be conducted to extract more nuanced perspectives on the needs of catchment water quality modelling such as with respect to different classes of problems. This would facilitate more in-depth analysis to support the more general findings from the survey.

\section{ACKNOWLEDGMENTS}

The Improving Source Constituent Modelling Collaborative Partnership, established in mid-2017, is a partnership between the Australian National University (ANU), State government agencies, the Commonwealth Scientific and Industrial Research Organisation (CSIRO), Alluvium and other water quality experts. We acknowledge the guidance and support provided by partners, particularly those who tested the pilot study or otherwise provided feedback on the survey questions. This work was funded by the Queensland Department of Environment and Science, Queensland Department of Natural Resources, Mines and Energy, Western Australia Department of Water and Environmental Regulation and New South Wales Department of Planning, Industry and Environment. Lastly, our appreciation goes out to all the survey respondents.

\section{REFERENCES}

Fu, B., Merritt, W. S., Croke, B. F. W., Weber, T. R., \& Jakeman, A. J. 2019. A review of catchment-scale water quality and erosion models and a synthesis of future prospects. Environmental Modelling \& Software, 114, 75-97.

Newham, L., Cuddy, S., Vertessy, R. \& Jakeman, A. 2004. Informing the design of catchment contaminant cycle modelling: a survey of end-user needs, CSIRO Land and Water. https://doi.org/10.4225/08/586be7f53c90c 